\title{
Percutaneous liver biopsy experience in a district general hospital
}

\author{
Author: Shuann Shwana
}

\section{Aims}

Percutaneous liver biopsy is the standard procedure for obtaining hepatic tissue for histopathological examination, and remains an essential tool in the diagnosis and management of parenchymal liver diseases. Our aim was to investigate the practice of liver biopsy in a small district general hospital.

\section{Methods}

A retrospective study of all liver biopsies that were carried out between 2008 and 2010 in a small district general hospital. This study describes the indications, complications and mortality of this procedure.

\section{Results}

Twenty-six patients ( 9 female and 17 male) had liver biopsies. Twenty-two patients had a clearly defined indication for the liver biopsy: abnormal liver function test (2), liver cirrhosis (3), autoimmune hepatitis (3), haemachromatosis (3), viral hepatitis (11), metastatic carcinoma (1), neuroendocrine tumour (1) and alpha-1 antitrypsin deficiency (2). All liver biopsies were ultrasoundguided and the appropriate blood tests were checked beforehand. Twenty-three biopsies were successful at the first attempt. All biopsies were a good size and led to 22 patients being discharged home after 6 hours. Pain was reported in six cases; otherwise, no complications or deaths were reported.

\section{Conclusions}

Percutaneous ultrasound-guided liver biopsy enables a good size and quality of sample to be taken in a safe and effective manner, with usually one pass being required with minimal associated complications. The results are comparable with other studies carried out in specialised hepatology centres.

\section{Conflict of interest statement}

None declared. 